\title{
Soft Handover in OFDMA based Visible Light Communication Networks
}

\author{
Ergin Dinc Ozgur Ergul Ozgur B. Akan \\ Next-generation and Wireless Communications Laboratory \\ Department of Electrical and Electronics Engineering \\ Koc University, Istanbul, 34450 Turkey. \\ Email: \{edinc, ozergul, akan\}@ku.edu.tr
}

\begin{abstract}
As the demand for wireless bandwidth rapidly increases, alternative methods to radio frequency-based communication are investigated to overcome the limited bandwidth problem. Visible light communication (VLC) using light emitting diodes (LEDs) is one of these alternatives. LEDs are estimated to replace the incandescent bulbs within the decade. Since, LEDs can be intensity modulated faster than the human eye can detect, illumination and communication can both be provided by the same lighting system. Indoors communication constitutes $70 \%$ of the overall traffic, and VLC is a promising technology to complement Wi-Fi and cellular wireless systems. However, proper handover mechanism should be developed for VLC to be a complete indoors solution. In this paper, we present two soft handover methods for VLC. Simulation results indicate our solutions provide higher data rate for both the overall system and individual users in the handover region.

Index Terms-Visible light, Handover, OFDMA, FFR
\end{abstract}

\section{INTRODUCTION}

High data rate applications such as high definition video streaming are being widely used. Meeting the required wireless bandwidth for these applications is one of the prominent challenges of new wireless communication systems. As a result, alternative wireless systems are being investigated. Visible light communication (VLC) is one of the alternatives.

LEDs offer various advantages such as lower cost, lower energy consumption and higher lifetime, compared to incandescent light bulbs widely used today. Therefore, LED based illumination is predicted to become the dominant lighting method in the near future. Since LEDs support high switching rates, it is possible to modulate the intensity of LED-generated light faster than human eye can perceive, enabling their use for communication as well as illumination. VLC using LEDs was first demonstrated at Nakagawa Laboratory, in 2003 [1]. Since then, high data rates up to $500 \mathrm{Mbps}$ were reported [2].

With such high data rates, VLC is envisioned to be used in high data rate indoors applications. Some of these applications include information dissemination in shopping malls for product information, and in museums for data on exposition pieces. In such applications, users move from one light source to the next. Meanwhile, their communication should not be effected by this switch over different light sources. Therefore, effective handover mechanisms for VLC are needed.

In this paper, we propose two different VLC handover mechanisms. We first show the need for such handover mechanisms quantitatively, on a scenario case. Then, we explain our two solution proposals. Finally, we present simulation results to show that our solutions increase both the overall system performance and the performance of each user.

The remainder of the paper is organized as follows. Section II provides the related works. Section III includes power calculations for VLC. In Section IV, we present our proposed handover algorithms. Section V includes simulation results. Lastly, the conclusions are presented in Section VI.

\section{RELATED WORK}

VLC is an alternative technique for wireless communication applications. Since VLC is intended to be utilized for especially high data rate applications, it requires high signalto-interference-plus-noise ratio (SINR) values. For this reason, most of the VLC application requires line of sight (LOS) paths. Therefore, VLC channels can be modeled with optical channel DC gain calculations with generalized Lambertian intensity to include direct LOS and first order reflected diffuse paths [1], [3].

Modulation bandwidth is one of most important challenges in VLC due to hardware imperfections. To alleviate effects of inter-symbol interference (ISI), orthogonal frequency-division multiplexing (OFDM) methods can be utilized for VLC [4][7] and high data rates can be maintained with the visible light. In [7], OFDM with quadrature-amplitude modulation is utilized to reach $513 \mathrm{Mbps}$. Thus, orthogonal frequencydivision multiple access (OFDMA) can be utilized as a multiple access scheme in VLC networks. [8], [9] introduce fractional frequency reuse (FFR) based resource allocation for VLC networks employing OFDMA. In this work, we propose soft handover algorithms to maintain high data rates under mobility.

Handover in VLC has certain differences compared to cellular systems. The serving area for a light source is considerably smaller in VLC. Moreover, since the directivity of visible light is higher than the radio waves, received power drops off more rapidly as users move away from the light source. Therefore, the handover region is much smaller. The challenge here is to act very quickly for successful handover. On the other hand, mobility rate is rather limited and precise position estimation via VLC is possible. This helps in predicting the handover need and being prepared before the user moves to handover region. One of the first studies on the subject handles 


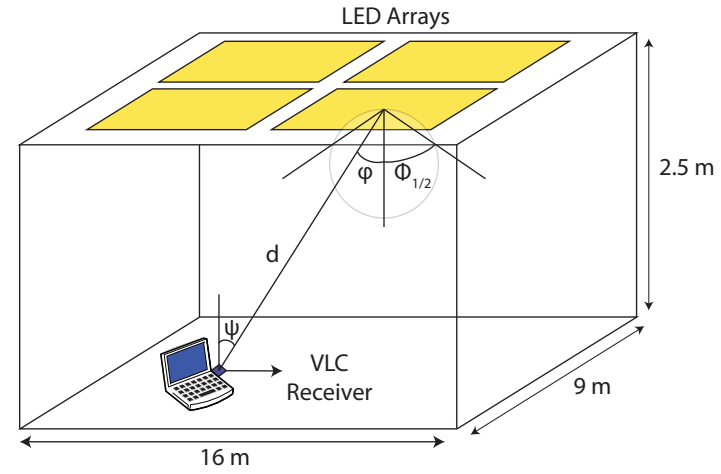

Fig. 1. VLC path and simulation room.

intensity based handover for VLC [10]. Other existing studies mostly focus on clustering and power adjustment methods for handover [8], handover protocols [11], extensions to IEEE 802.15.7 for handover support [12], and cell zooming methods [13]. RF-hybrid systems that utilize Wi-Fi when a VLC link is not available have also been considered [14]. However, to the best of our knowledge, there is no solution for soft handover specifically tailored to VLC.

\section{SYSTEM MODEL}

In this section, we will first introduce power calculations for the visible light and fractional frequency reuse (FFR) in VLC networks.

\section{A. Channel Model}

In VLC, information exchange is performed via light. Since the wavelength of visible light is much smaller than photodetector (PD) area, phase of the light cannot be modulated. Therefore, transmitted signal in VLC is required to be positive and real. [15]. In addition, light intensity strongly depends on distance. Thus, multi-path components will be considerably lower except near walls. Since the main aim of the paper is to show the performance of handover algorithms, we only consider LOS paths as in [8].

Since transmitted signals in VLC are real and positive, relationship between transmit power and receive power can be directly calculated with channel DC gain $(H(0))$ [3]. Therefore, the received power can be found as

$$
P_{r}=H(0) P_{t},
$$

where $P_{r}$ is the received power and $P_{t}$ is the transmit power.

Several LOS components can be detected by an optical receiver. Thus, we need to calculate sum of these components. For this purpose, we utilize generalized Lambertian intensity [16]. For the path shown in Fig. 1, DC gain of the optical link can be found as [1], [3]

$$
H(0)= \begin{cases}\frac{(m+1) A}{2 \pi d^{2}} \cos ^{m}(\phi) T_{s} g(\psi) \cos (\psi), & 0 \leq \psi \leq \psi_{c} \\ 0, & \psi>\psi_{c}\end{cases}
$$

where $m=-\ln (2) / \ln \left(\cos \left(\Phi_{1 / 2}\right)\right)$ where $\Phi_{1 / 2}$ is the transmitter semi-power angle, $d$ is the distance between LED and $\mathrm{PD}, \phi$ is the angle of irradiance at $\mathrm{PD}, \psi$ is the angle of incidence at transmitter LED, $T_{s}$ is the gain of the optical filter, $A$ is the area of the detector, $\psi_{c}$ is the width of receiver field of vision and $g(\psi)$ is the optical concentrator gain given as

$$
g(\psi)= \begin{cases}\frac{n^{2}}{\sin ^{2}\left(\psi_{c}\right)}, & 0 \leq \psi \leq \psi_{c} \\ 0, & \psi>\psi_{c}\end{cases}
$$

where $n$ is the refractive index of the air.

\section{B. FFR for $V L C$}

VLC communication is provided via single LOS path in general. However, inter-symbol interference (ISI) is still a limiting factor in VLC due to the hardware imperfections [17]. Since OFDM is promising in order to eliminate ISI, orthogonal frequency division multiple access can be utilized as multiple access scheme. FFR schemes are introduced for VLC in [8], [9]. Fig. 2 presents FFR employment with reuse factor of 3.

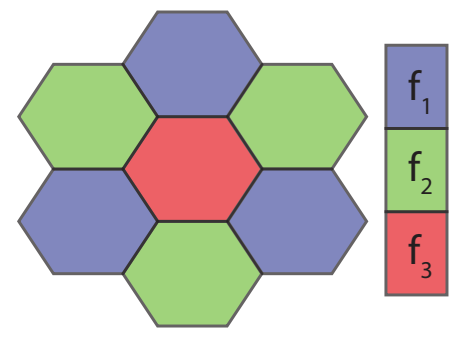

Fig. 2. FFR employment.

Since overall frequency spectrum is divided into many subchannels in OFDMA, each sub-channel can be assumed as flat. Therefore, received signal-to-interference plus noise ratio

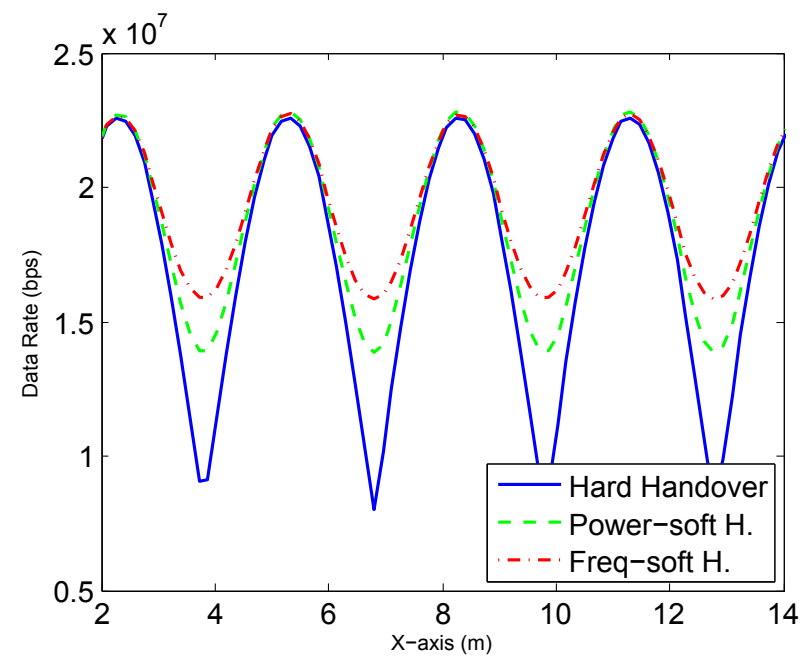

Fig. 3. Handover location. 


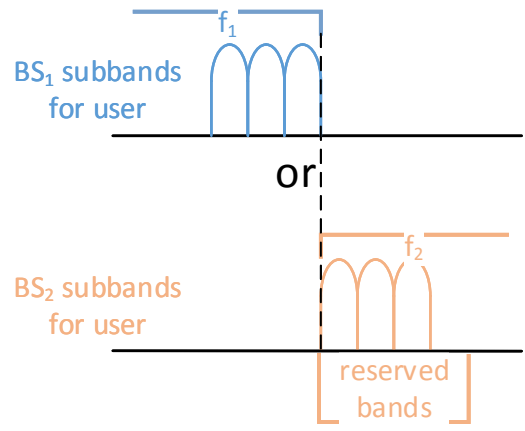

(a) Hard handover.

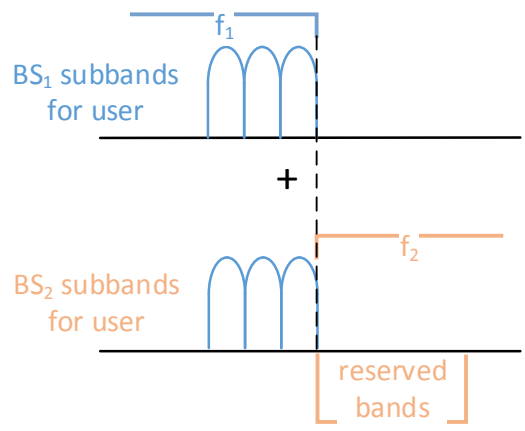

(b) Power-based.

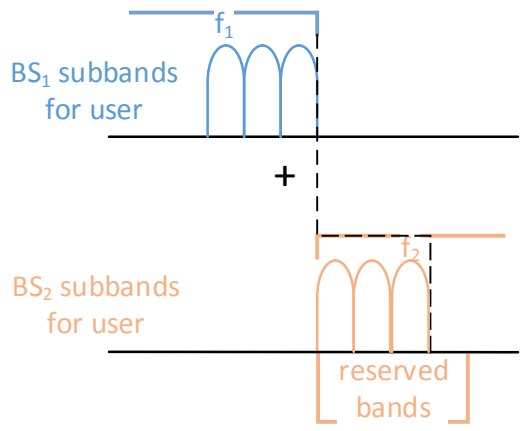

(c) Frequency-based.

Fig. 4. Use of sub-bands during handover

(SINR) for $j$ th sub-channel can be found as

$$
S I N R_{j}=\frac{\left(\sum_{i=\mathbf{S}} \rho H_{i, j, n} P_{i, j}\right)^{2}}{\left(\sum_{k=\mathbf{I}_{j}} \rho H_{k, j} P_{k, j}\right)^{2}+\sigma^{2}},
$$

where $\mathbf{S}$ is the serving stations, $\mathbf{I}_{j}$ is the interfering stations for sub-channel $j, \rho$ is responsivity of PD, $P_{i, j}$ power of transmitter array $i$ for sub-channel $j, H_{i, j}$ is the channel DC gain between transmitter $i$ and user $n, \sigma^{2}$ is the noise power due to mainly shot noise [1], [3].

\section{HANDover Algorithms}

The received power in VLC changes considerably with PD irradiance angle, $\phi$ and LED incidence angle, $\psi$ as seen in Eqn. (2). Therefore, as users move, their data rates will fluctuate. We depict this in Fig. 3. The straight line shows the rate experienced by the user as he moves, when hard handover method is used.

In the hard handover method, the user is served by one source at a time. As the user moves from one cell to the other, the received power from the base station (BS) (in our case the light source) goes below a threshold value. Then, the user drops its connection with this BS and connects to another BS. As seen in Fig. 3, the rate fluctuation is high for hard handover. To alleviate this, soft handover methods are used. In a soft handover scheme, the user is served by two BSs when he is in the intersection area, therefore, his data rate does not drop off suddenly.

The knowledge of user location is important for efficient and effective handover. VLC offers an advantage in this front, since precise position estimation is possible using VLC [18]. This is fortunate, since received power fluctuate much more rapidly compared to RF. In VLC, cell radius is in the order of meters, whereas in cellular communication, it is in the order of hundreds of meters even for picocells. In our solution we assume the position of the user is known. Since position estimation algorithms are out of the scope of this paper, we do not go into details of a position estimation scheme. For example, the method proposed in [18] may be used. Once the position of the user can be tracked, we know to which BS the user will be handed over to. In our solution, we assume we have this information.

We propose two soft handover solutions. Both methods assume the FFR scheme depicted in Fig. 2. However, for handover to succeed, each BS should reserve a certain percentage of its sub-bands for users arriving from neighbor cells. Otherwise, the BS can use all of its resources on its existing users and cannot serve incoming users. Therefore, we assume, each BS reserves a certain percentage of its sub-bands for handover.

At first, assume the user is under a certain BS that uses frequency range $f_{1}$, and is served by that BS. The user starts moving towards a BS operating under $f_{2}$, and as the incidence angle increases, the received power and therefore the signal to interference plus noise ratio (SINR) drops below a certain threshold value. Since location information of the user is known, the received power, interference and SINR can be calculated for each user. Therefore, each BS knows when a certain user is above its SINR threshold value and starts serving the user. When the user has an SINR above threshold for two BSs, we say the user is in the intersection area. Below, we detail our two proposals under these assumptions.

The first method is called the power-based soft handover. As the user moves into the intersection area of two BS, the

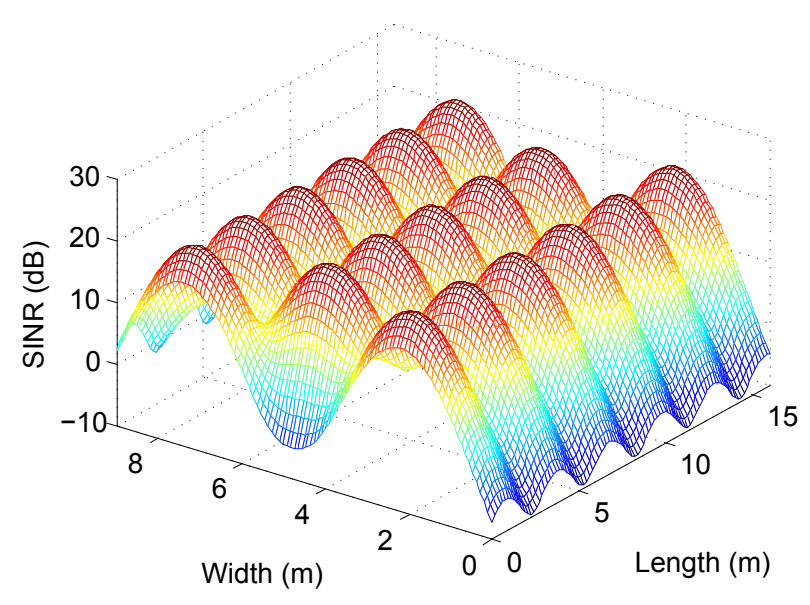

Fig. 5. Handover location. 


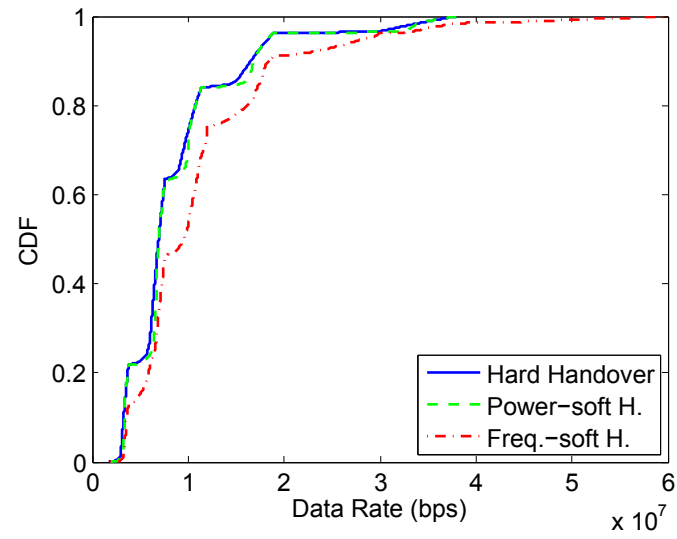

(a) All cell users $\beta=0.5$.

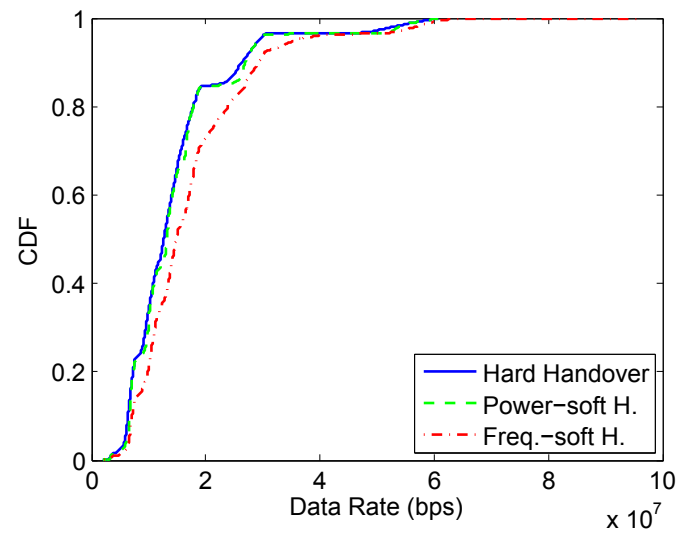

(c) All cell users $\beta=0.8$.

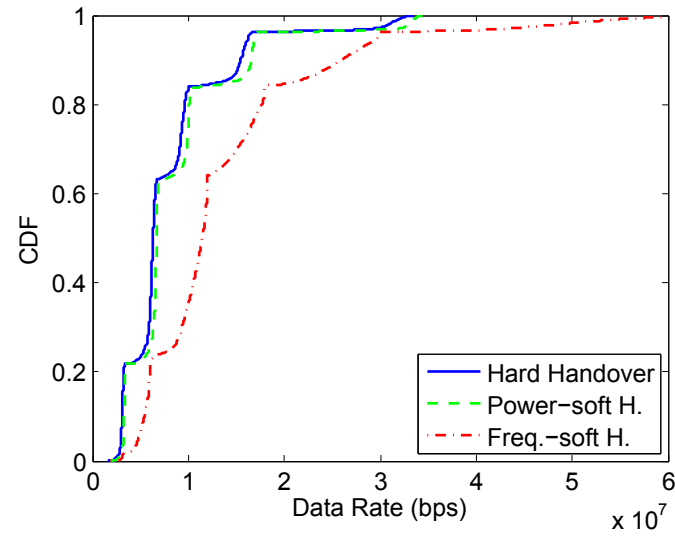

(b) Cell edge users $\beta=0.5$.

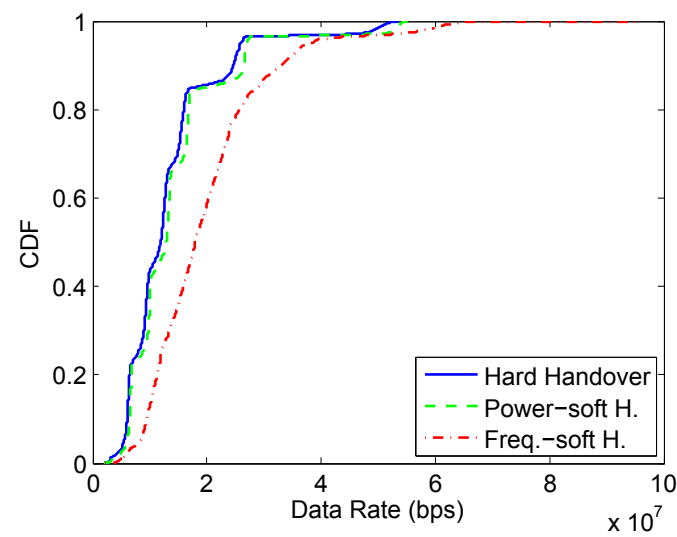

(d) Cell edge users $\beta=0.8$.

Fig. 6. CDF of data rates for soft handover algorithms

second BS starts sending the same data the first BS sends using the same frequency range that the first BS uses. The two intensities will be combined in the receiver, increasing the SINR. This is only possible in VLC, where multi-path from two different transmitters does not have an adverse effect on the receiver. This is due to the fact that the wavelength of visible light is much smaller than the photo diode surface area. Therefore, there is no destructive combining at the receiver due to the phase difference between the two paths. We show the change of data rate as the user moves with dashed line in Fig. 3 . As seen here, power-based soft handover causes less rate fluctuation compared to hard handover.

The second method is called the frequency-based soft handover. In this scheme, when the user goes into the intersection area, the second BS starts serving it using some of its subbands reserved for handover. The SINR from both stations will be lower at the intersection area, however, this will be compensated by the additional bandwidth provided to the user. Frequency-based soft handover data rate is show with the dash-dot line in Fig. 3. This method also reduces the data rate fluctuation considerably, compared to the hard handover method.

The idea of the proposed schemes is depicted in Fig. 4. The figure shows the use of sub-bands in the intersection area.
In the hard handover, the user either uses the sub-bands of the closest BS. In the power-based soft handover, the second BS starts transmitting the same sub-bands as the first BS in the intersection area. The frequency-based soft handover, the second BS increases the bandwidth of the user by starting transmission in its reserved bands.

\section{Simulation Results}

In this section, we provide simulation results to justify our claims. Table I includes the simulation parameters. We perform our simulations for $16 \times 9 \times 2.5 \mathrm{~m}^{3}$ room as in Fig. 1. VLC network utilizes FFR with reuse factor 3 as shown in Fig. 2. The user locations are determined randomly with uniform distribution on MATLAB and we perform 100000 iterations for each simulation.

In Fig. 5, we present SINR results for modeled VLC network employing FFR. As noticed, SINR at cell edges become very low. Therefore, if a user moves towards to cell edge, the data rate of the user will be considerable lower. This is the main reason for the proposed soft handover algorithms.

We present the cumulative distribution function (CDF) of user data rates under different handover algorithms for VLC in Fig. 6. In order to make a handover, neighboring cell is required to have empty sub-channels to assign. We control the 
TABLE I

SIMULATION PARAMETERS.

\begin{tabular}{|l|c|c|}
\hline \multicolumn{3}{|c|}{ Transmitter Parameters } \\
\hline Parameter & Value & Unit \\
\hline Transmitter optical power $\left(P_{t}\right)$ & 63 & $\mathrm{~mW}$ \\
\hline Semi-angle half power $\left(\Phi_{1 / 2}\right)$ & 70 & Degree \\
\hline \# of LEDs & $60 \times 60$ & - \\
\hline LED interval & 0.01 & $\mathrm{~m}$ \\
\hline Distance between LED arrays & 3 & $\mathrm{~m}$ \\
\hline \multicolumn{2}{|c|}{ Receiver Parameters } \\
\hline Receiver FOV $\left(\psi_{c}\right)$ & 60 & Degree \\
\hline PD area $(\mathrm{A})$ & 1 & $\mathrm{~cm}^{2}$ \\
\hline Optical filter gain $\left(T_{s}\right)$ & 1 & - \\
\hline Conversion efficiency $(\rho)$ & 0.53 & $\mathrm{~A} / \mathrm{W}$ \\
\hline \multicolumn{2}{|c|}{ System Parameters } \\
\hline System bandwidth $\left(B_{s y s}\right)$ & 20 & $\mathrm{MHz}$ \\
\hline \# of subcarriers & 512 & - \\
\hline FFR reuse factor & 3 & - \\
\hline \# of users & 33 & - \\
\hline Noise density $\left(N_{0}\right)$ & $1 \times 10^{-21}$ & $\mathrm{~A}^{2} / \mathrm{Hz}$ \\
\hline
\end{tabular}

amount of handover sub-channels with $\beta$ which represents the ratio of assigned sub-channels to overall sub-channels that a cell have.

In Fig. 6(a) and 6(b), we present CDF of user data rates in the middle cell for $\beta=0.5$. As noticed, frequencysoft handover outperforms other methods in terms of edge user data rates. As $\beta$ decreases, the performance increase becomes higher because the amount of sub-channels available increase for the frequency-soft handover. However, power-soft handover is not affected by changing $\beta$ because it does not require additional sub-channels. Since there will be less subchannels to assign for low $\beta$ values, user data rates becomes lower for lower $\beta$ values as noticed in Fig. 6. To sum up, frequency-soft handover has higher chance of providing higher data rates.

\section{CONCLUSION}

VLC is a promising communication method for high data rate applications especially in indoor environments. In this study, we propose two soft handover methods for VLC to improve the performance of the users at the cell boundaries. According to our results, our proposed handover methods improve the user data rates. Especially, frequency-soft handover outperforms both power-soft and hard handover.

\section{ACKNOWLEDGMENT}

This work was supported by TURK TELEKOM.

\section{REFERENCES}

[1] T. Komine, M. Nakagawa, "Fundamental analysis for visible-light communication system using LED lights," IEEE Transactions on Consumer Electronics, vol. 50, no. 1, Feb. 2004, pp.100-107, doi: 10.1109/TCE.2004.1277847.

[2] "500 Megabits/Second with White LED Light," (Press release) Siemens. Jan 18, 2010. Available at http://www.siemens.com/innovation/en/news/2010/500-megabitssecondwith-white-led-light.htm

[3] J. M. Kahn, J. R. Barry, "Wireless infrared communications," Proceedings of the IEEE, vol. 85, no. 2, Feb 1997, pp. 265-298.
[4] A. Shaw, A. Bradley, J. Donegan, and J. Lunney, "GaN resonant cavity light-emitting diodes for plastic optical fiber applications," IEEE Photon. Technol. Lett., vol. 16, no. 9, Sep. 2004, pp. 2006-2008.

[5] Y. Tanaka, T. Komine, S. Haruyama, M. Nakagawa, "Indoor visible communication utilizing plural white LEDs as lighting," in: IEEE International Symposium on Personal, Indoor and Mobile Radio Communications (PIMRC), San Diego, CA, Sept. 2001, pp. F81-F85.

[6] M. Z. Afgani, H. Haas, H. Elgala, D. Knipp, "Visible light communication using OFDM," International Conference on Testbeds and Research Infrastructures for the Development of Networks and Communities (TRIDENTCOM), 2006.

[7] J. Vucic, C. Kottke, S. Nerreter, K.-D. Langer, J. W. Walewski, "513 Mbit/s Visible Light Communications Link Based on DMT-Modulation of a White LED," Journal of Lightwave Technology, vol. 28, no. 24, Dec.15, 2010, pp. 3512-3518.

[8] C. Chen, N. Serafimovski, H. Haas, "Fractional frequency reuse in optical wireless cellular networks," in Proc. IEEE PIMRC'13, pp.3594-3598, 811 Sept. 2013.

[9] B. Ghimire, H. Haas, "Resource Allocation in Optical Wireless Networks," in Proc. of the 22nd Annual IEEE International Symposium on Personal, Indoor and Mobile Radio Communications (PIMRC 11), 1114 Sep. 2011.

[10] A. M. Vegni, T. D. C. Little, "Handover in VLC Systems with Cooperating Mobile Devices," in Proc. Mobility and Communication for Cooperation and Coordination workshop, part of the IEEE Intl. Conf. on Computing, Networking and Communications (ICNC 2012), January 30 - February 2, 2012, Maui, Hawaii, USA.

[11] X. Bao, X. Zhu, T. Song, Y. Ou, "Protocol Design and Capacity Analysis in Hybrid Network of Visible Light Communication and OFDMA Systems," Vehicular Technology, IEEE Transactions on , vol.63, no.4, pp. 1770,1778, May 2014

[12] T. Nguyen, Y. M. Jang, M. Z. Chowdhury, "A pre-scanning-based link switching scheme in visible light communication networks," Ubiquitous and Future Networks (ICUFN), 2013 Fifth International Conference on vol., no., pp.366,369, 2-5 July 2013

[13] M. B. Rahaim, T.D.C. Little, "SINR analysis and cell zooming with constant illumination for indoor VLC networks," Optical Wireless Communications (IWOW), 2013 2nd International Workshop on , vol., no., pp.20,24, 21-21 Oct. 2013

[14] M.B. Rahaim, A. M. Vegni, T.D.C. Little, "A hybrid Radio Frequency and broadcast Visible Light Communication system," GLOBECOM Workshops (GC Wkshps), 2011 IEEE , vol., no., pp.792,796, 5-9 Dec. 2011

[15] D. Tsonev, S. Videv, H. Haas, "Light fidelity (Li-Fi): towards all-optical networking," in: Proc. SPIE 9007, Broadband Access Communication Technologies VIII, 900702 (December 18, 2013); doi: $10.1117 / 12.2044649$.

[16] F. R. Gfeller, U. H. Bapst, "Wireless in-house data communication via diffuse infrared radiation," in Proc. IEEE, vol. 67, Nov. 1979, pp. 14741486.

[17] Chaopei Wu, Hua Zhang, Wei Xu, "On visible light communication using LED array with DFT-Spread OFDM," IEEE International Conference on Communications (ICC), 10-14 June 2014, pp. 3325-3330.

[18] S-H. Yang, E-M. Jung, S-K. Han, "Indoor Location Estimation Based on LED Visible Light Communication Using Multiple Optical Receivers," IEEE Communications Letters, vol.17, no.9, pp.1834,1837, September 2013 